\title{
Obstetrician and Gynecologist Utilization of the Noninvasive Prenatal Testing Expanded Option
}

\author{
Sarah Mayes, MS, $\mathrm{CGC}^{1}$ Syed Hashmi, MD, MPH, $\mathrm{PhD}^{2}$ Mark A. Turrentine, $\mathrm{MD}^{3}$ \\ Sandra Darilek, MS, CGC ${ }^{4}$ Lara A. Friel, MD, PhD ${ }^{5}$ Jennifer Czerwinski, MS, CGC ${ }^{5}$ \\ ${ }^{1}$ Genetic Counseling Program, University of Texas Graduate School of \\ Biomedical Science at Houston, Houston, Texas \\ 2 Department of Pediatrics, The University of Health Science Center at \\ Houston, Houston, Texas \\ ${ }^{3}$ Department of Obstetrics and Gynecology, Kelsey-Seybold Clinic, \\ Houston, Texas \\ ${ }^{4}$ Department of Molecular and Human Genetics, Baylor College of \\ Medicine, Houston, Texas \\ ${ }^{5}$ Department of Obstetrics, Gynecology, and Reproductive Sciences, \\ The University of Texas Health Science Center at Houston, \\ Houston, Texas \\ Address for correspondence Sarah Mayes, MS, CGC, Department of \\ Molecular and Human Genetics, Baylor College of Medicine, 7900 \\ Fannin Suite 2790, Houston, TX 77054 \\ (e-mail: smayes@bcm.edu). \\ Am J Perinatol Rep 2016;6:e18-e24.
}

\begin{abstract}
Keywords

- noninvasive prenatal testing

- microdeletion

- prenatal screen

Objective Noninvasive prenatal testing (NIPT) enables the detection of common fetal aneuploidies such as trisomy 21 , trisomy 18 , trisomy 13 , and sex chromosome abnormalities via analysis of cell-free fetal DNA circulating in maternal serum. In October 2013, the option to screen for additional trisomies and select microdeletion syndromes became clinically available. The complex testing methods, oftentimes unclear clinical utility of results, and lack of professional guidelines renders it challenging for clinicians to keep abreast of evolving prenatal screening options. We undertook a survey to assess physicians' awareness of, utilization of, and attitudes toward the expanded NIPT option.

Study Design Obstetricians attending hospital service meetings in the Houston Texas Medical Center completed an anonymous survey regarding the utilization patterns of expanded NIPT.

Results Overall, 85 obstetricians were surveyed. While all respondents indicated awareness of NIPT in its traditional form, 75\% (64/85) were aware of the expanded testing option, and $14 \%(12 / 85)$ reported having ordered the expanded NIPT option. A total of $91 \%(77 / 85)$ expressed that practitioners need more information regarding the screening.

Conclusion Based on these findings and the fluid landscape of prenatal screening, education, and reeducation of health care professionals is imperative to ensure responsible patient counseling, informed consent, and appropriate posttest management.
\end{abstract}

received

July 6, 2015

accepted after revision

August 15, 2015

published online

October 29, 2015
DOI http://dx.doi.org/

$10.1055 / \mathrm{s}-0035-1566313$.

ISSN 2157-6998.
Copyright $\odot 2016$ by Thieme Medical

Publishers, Inc., 333 Seventh Avenue,

New York, NY 10001, USA.

Tel: +1(212) 584-4662.
License terms

(c) (i) $\ominus$ (\$) 


\section{Background}

Since it first became clinically available in October 2011, noninvasive prenatal testing (NIPT) has been well studied. It has been shown to have high detection rates as well as significantly lower false-positive rates, and higher positive predictive values for detection of trisomy 21 and trisomy 18 when compared with alternative screening options. ${ }^{1-7}$ NIPT has been widely accepted by professional societies as a clinically valid prenatal screen when utilized with good clinical judgment and proper informed consent. ${ }^{8-11}$

In October 2013, the option to screen for several additional conditions, including other select trisomies and specific syndromes caused by chromosomal microdeletions, such as 22q deletion syndrome (DiGeorge syndrome) and 5p-syndrome (Cri-du-chat syndrome) became available. As of June 2015, there have been no large, prospective studies that address the ability of this technology to detect these additional trisomies and microdeletion syndromes in maternal serum. Several proof of concept studies have demonstrated the technology's ability to detect subchromosomal abnormalities, yet the most comprehensive study to date estimates positive predictive values for the examined microdeletion syndromes ranging from 3.8 to $17.0 \%{ }^{12-19}$ While this new expanded testing option is currently clinically available and easily accessible, the accuracy and precision of the testing as well as the correct interpretation of results remain uncertain. This gap between available information and informed interpretation presents physicians and other health care providers with the difficult decision of whether to offer this testing to patients and how to clinically utilize results with little published guidance from professional societies. ${ }^{20,21}$ It is concerning that physicians may be more inclined to order the expanded NIPT option as a result of laboratory marketing, possibly without having the opportunity to appreciate the complex nuances of expanded NIPT. The objective of this study was to characterize current practices among obstetricians regarding the expanded NIPT option to determine whether any education is needed or desired by those implementing this new testing opportunity in their practices.

\section{Methods}

Eligibility included physicians whose obstetrical departments were affiliated with the genetic counseling program at the University of Texas Graduate School of Biomedical Science at Houston. Physicians who attended Houston area obstetrical departmental meetings were surveyed from September 2014 to February 2015. To be included in this study, respondents must have indicated their primary practice setting was as a specialist in obstetrics and gynecology. All participants were completely trained and beyond their residency. Information was collected by an anonymous survey, which contained three sections: demographic information, assessment of awareness of the expanded NIPT option, and description of obstetrician's attitudes toward the expanded NIPT option. The survey was distributed at 12 clinic sites located at 4 institutions (Memorial Hermann, University of
Texas Health, Baylor College of Medicine, and Kelsey-Seybold Clinic).

Data collected from the surveys was analyzed for all applicable variables using the statistical analysis software program, STATA (v.13.0, College Station, TX). Descriptive charts and graphs were created using Microsoft Excel (Microsoft Corp., Redmond, WA). A comparison between groups was performed using contingency tests (Chi-square analysis or Fisher exact test) or Kruskal-Wallis tests where appropriate. Statistical significance was assumed if $p<0.05$.

\section{Results}

A total of 118 surveys were distributed, of which $78 \%(92 / 118)$ were returned. Seven individuals did not meet the inclusion criteria of practicing primarily in Obstetrics and Gynecology and were excluded from all analyses. Therefore, the final sample size for analysis was 85 . The survey queries were selective in terms of which respondents were eligible to answer each question, which resulted in varying numbers of total responses. See $\boldsymbol{-}$ Table $\mathbf{1}$ for complete demographic data. The majority $80 \%(68 / 85)$ reported practicing in a private setting. The median time participants reported practicing in the indicated setting was 12 years (range, $0.13-40$ years).

\section{NIPT and Expanded NIPT Knowledge and Awareness}

A summary of participants' clinical utilization of NIPT is included in - Table 2. All participants indicated that they were aware of NIPT, with 75\% (64/85) reporting that they were aware of the expanded testing option of NIPT. The majority of those who have ordered NIPT reported that

Table 1 Demographics of eligible respondents $(n=85)$

\begin{tabular}{|l|l|}
\hline Demographics & $\boldsymbol{n}(\%)$ \\
\hline Gender & $35(41)$ \\
\hline Male & $49(58)$ \\
\hline Female & $1(1)$ \\
\hline No response & $49(58)$ \\
\hline Race & $10(12)$ \\
\hline White (non-Hispanic) & $8(9)$ \\
\hline Hispanic or Latino & $12(14)$ \\
\hline Black or African American & $5(6)$ \\
\hline Asian or Pacific Islander & $1(1)$ \\
\hline Other & \\
\hline No response & $68(80)$ \\
\hline Primary practice setting & $14(16)$ \\
\hline Private practice & $3(4)$ \\
\hline Academic/University Medical Center &
\end{tabular}


e20 Obstetrician and Gynecologist Utilization of the NIPT Expanded Option Mayes et al.

Table 2 Obstetrician responses to questions about clinical utilization of NIPT

\begin{tabular}{|c|c|}
\hline Responses & $n(\%)$ \\
\hline \multicolumn{2}{|l|}{ Before today, were you aware of the expanded testing option of NIPT? $(n=85)$} \\
\hline Yes & $64(75)$ \\
\hline No & $12(14)$ \\
\hline I am unsure & $9(11)$ \\
\hline \multicolumn{2}{|l|}{ If yes, how did you learn about expanded testing? Check all that apply. $(n=64)$} \\
\hline Medical literature & $31(48)$ \\
\hline Professional society/conference & $15(23)$ \\
\hline Colleague & $10(16)$ \\
\hline Marketing from laboratories that offer it & $30(47)$ \\
\hline Educational lecture & $12(19)$ \\
\hline Other & $2(3)$ \\
\hline \multicolumn{2}{|l|}{ If you order NIPT, what laboratory do you order from? Check all that apply. $(n=69)$} \\
\hline Sequenom/MaterniT21 plus & $34(49)$ \\
\hline Natera/Panorama & $26(38)$ \\
\hline Verinata/Verifi/Illumina & $20(29)$ \\
\hline Ariosa/Labcorp/Harmony & $30(44)$ \\
\hline Other & $7(10)$ \\
\hline I am unsure & $4(6)$ \\
\hline It depends on & $10(15)$ \\
\hline No response & $1(2)$ \\
\hline \multicolumn{2}{|l|}{ For which patients do you order/refer for NIPT? Check all that apply. $(n=85)$} \\
\hline All patients at increased risk for fetal aneuploidy (AMA, ultrasound finding, etc.) & $50(59)$ \\
\hline Only patients at increased risk for fetal aneuploidy who decline invasive testing & $15(18)$ \\
\hline Patients who request it & $28(33)$ \\
\hline All pregnant patients & $13(15)$ \\
\hline Other & $3(4)$ \\
\hline No response & $12(14)$ \\
\hline \multicolumn{2}{|c|}{ If a patient had a positive NIPT result, what would be your first recommendation? Please select the best answer. $(n=69)$} \\
\hline I would offer CVS/amniocentesis & $22(32)$ \\
\hline I would recommend a targeted ultrasound & $2(3)$ \\
\hline I would refer the patient to a specialist, such as an MFM or genetic counselor & $49(71)$ \\
\hline Other & $2(3)$ \\
\hline
\end{tabular}

Abbreviations: AMA, advanced maternal age; CVS, chorionic villus sampling; MFM, maternal-fetal medicine; NIPT, noninvasive prenatal testing.

they were aware of the expanded testing option of NIPT (80\%, 55/69). This is compared with those who did not report having ordered NIPT, of whom only 64\% (7/11) indicated that they were aware of the expanded testing option $(p=0.05)$. The majority of participants $(82 \%, 69 / 85)$ indicated having ordered NIPT and an additional 5\% (4/85) responded that they always refer to a maternal-fetal medicine subspecialist (MFM) or genetic counselor for NIPT. The median number of NIPT orders per month was 4 (range, 1-30). Just under half of the participants $(49 \%, 35 / 71)$ reported never seeing a positive result. Among the remaining participants, about half $(51 \%, 19 / 37)$ had seen only one positive result with nearly all $(97 \%, 36 / 37)$ having seen not more than five positive results. Overall, obstetricians report making appropriate recommendations following a positive NIPT result by offering diagnostic testing (32\%, 22/69) and/or referrals to subspecialists $(71 \%, 49 / 69)$.

A total of 12 individuals $(17 \%, 12 / 69)$ reported that they order the expanded testing option when ordering NIPT. Only one participant reported having seen a positive expanded NIPT result. - Table 3 contains a summary of the clinical utilization of expanded NIPT. When asked what factors are used when determining when to offer expanded NIPT, the most common response was insurance coverage/consideration of whether 
Table 3 Obstetrician responses to questions about clinical utilization of expanded NIPT

\begin{tabular}{|c|c|}
\hline Responses & $n(\%)$ \\
\hline \multicolumn{2}{|c|}{ For which patients do you order the expanded testing option when ordering NIPT? Check all that apply. $(n=12)$} \\
\hline Family history of trisomy 16 , trisomy 22 , or a microdeletion syndrome & $7(58)$ \\
\hline Ultrasound indicative of trisomy 16 , trisomy 22 , or a microdeletion syndrome & $5(42)$ \\
\hline Those who request it & $6(50)$ \\
\hline All pregnant patients who pursue NIPT & $4(33)$ \\
\hline \multicolumn{2}{|c|}{$\begin{array}{l}\text { For those patients for whom you do not order the expanded testing option, what factors influence your decision not to order it? } \\
\text { Check all that apply. }(n=12)\end{array}$} \\
\hline Lack of interest from my patient & $3(25)$ \\
\hline My patient cannot afford it/it is not covered by their insurance & $7(58)$ \\
\hline Not enough published data regarding accuracy & $1(8)$ \\
\hline My professional society(ies) have not published guidelines & $1(8)$ \\
\hline I order this testing for all of my patients & $2(17)$ \\
\hline Other & $1(8)$ \\
\hline No response & $2(17)$ \\
\hline \multicolumn{2}{|c|}{$\begin{array}{l}\text { If a patient had a positive NIPT expanded testing result, what would be your first recommendation? Please select the best answer. } \\
(n=12)\end{array}$} \\
\hline I would offer CVS/amniocentesis & $3(25)$ \\
\hline I would refer the patient to a specialist, such as an MFM or genetic counselor & $10(83)$ \\
\hline \multicolumn{2}{|c|}{ With regard to the NIPT expanded testing option, when do you refer patients to an MFM/genetic counselor? $(n=29)$} \\
\hline Before ordering the test & $13(45)$ \\
\hline Only when patients have a positive result & $16(55)$ \\
\hline I am unsure & $2(7)$ \\
\hline \multicolumn{2}{|c|}{ What do you tell your patients the accuracy is for the NIPT expanded testing option? $(n=12)$} \\
\hline $99-100 \%$ & $6(50)$ \\
\hline $90-98 \%$ & $3(25)$ \\
\hline I am unsure & $2(17)$ \\
\hline No response & $1(8)$ \\
\hline
\end{tabular}

Abbreviations: CVS, chorionic villus sampling; MFM, maternal-fetal medicine; NIPT, noninvasive prenatal testing.

the patient can afford it (58\%, 7/12). Respondents who order expanded NIPT tended to refer to a subspecialist, such as an MFM or genetic counselor, only after receiving a positive result $(83 \%, 10 / 12)$ rather than before ordering $(17 \%, 2 / 12)$. Finally, half $(50 \%, 6 / 12)$ of the respondents who do order expanded NIPT report that they tell patients that the testing is 99 to $100 \%$ accurate. No respondents selected accuracies lower than $90 \%$ or indicated that they do not tell patients this information. Participants were also asked about their perceptions of expanded NIPT (-Table 4). Although, no professional guidelines concerning the expanded NIPT option had yet been published, 31\% (20/64) indicated that they were aware of professional guidelines for the expanded NIPT option. Over two-thirds $(68 \%, 58 / 85)$ of respondents indicated that they were unsure whether their laboratory of choice offers expanded NIPT in an opt-in or opt-out format. Finally, $41 \%$ (35/85) reported that they either currently order expanded NIPT or plan to incorporate expanded NIPT into their practices in the future.

\section{Attitudes toward Expanded NIPT}

Participants were asked to share their attitudes toward expanded NIPT by indicating their comfort level, explaining the testing to patients using a Likert scale ranging from very uncomfortable to very comfortable, and by selecting whether they consider expanded testing a screening or a diagnostic test (-Table 5). Participants were divided into those who reported ordering the expanded testing option of NIPT and those who did not. Of those who did not report ordering expanded NIPT, only 34\% (25/73) indicated that they felt at least somewhat comfortable explaining the testing to patients, whereas the majority $(83 \%, 10 / 12)$ of those who reported ordering expanded NIPT indicated that they felt at least somewhat comfortable explaining it to patients $(p=0.012)$. In addition, $14 \%(10 / 73)$ of those who reported that they do not order the expanded testing option identified it as a diagnostic test, compared with $33 \%(4 / 12)$ of those who reported that they have ordered expanded NIPT $(p<0.01)$. 
e22 Obstetrician and Gynecologist Utilization of the NIPT Expanded Option Mayes et al.

Table 4 Obstetrician responses to questions about perceptions of expanded NIPT

\begin{tabular}{|c|c|}
\hline Responses & $n(\%)$ \\
\hline \multicolumn{2}{|c|}{$\begin{array}{l}\text { Are you aware of professional guidelines concerning the } \\
\text { expanded NIPT option? }(n=64)\end{array}$} \\
\hline Yes & $20(31)$ \\
\hline No & $19(30)$ \\
\hline I am unsure & $18(28)$ \\
\hline No response & $7(11)$ \\
\hline \multicolumn{2}{|c|}{$\begin{array}{l}\text { Does your laboratory of choice offer the expanded testing } \\
\text { option in the opt-in or opt-out format? }(n=73)\end{array}$} \\
\hline Opt-in & $19(26)$ \\
\hline Opt-out & $2(3)$ \\
\hline I am unsure & $50(68)$ \\
\hline No response & $2(3)$ \\
\hline \multicolumn{2}{|c|}{$\begin{array}{l}\text { Do you plan on incorporating the NIPT expanded testing } \\
\text { option into your practice in the future? }(n=85)\end{array}$} \\
\hline Yes & $24(28)$ \\
\hline I currently order NIPT expanded testing & $11(13)$ \\
\hline I am unsure & $42(50)$ \\
\hline No & $8(9)$ \\
\hline
\end{tabular}

Abbreviation: NIPT, noninvasive prenatal testing.

Table 5 Obstetrician responses to questions about comfort with expanded NIPT $(n=85)$

\begin{tabular}{|c|c|}
\hline Responses & $n(\%)$ \\
\hline \multicolumn{2}{|c|}{$\begin{array}{l}\text { How comfortable are you with explaining the expanded } \\
\text { testing option of NIPT to your patients? }\end{array}$} \\
\hline Very uncomfortable & $10(12)$ \\
\hline Somewhat uncomfortable & $23(27)$ \\
\hline Neutral & $13(15)$ \\
\hline Somewhat comfortable & $26(30)$ \\
\hline Very comfortable & $9(11)$ \\
\hline No response & $4(5)$ \\
\hline \multicolumn{2}{|c|}{$\begin{array}{l}\text { Which do you consider the NIPT expanded testing option to } \\
\text { be? }\end{array}$} \\
\hline A screen & $58(68)$ \\
\hline A diagnostic test & $14(17)$ \\
\hline Neither & $1(1)$ \\
\hline I am unsure & $10(12)$ \\
\hline No response & $2(2)$ \\
\hline
\end{tabular}

Abbreviation: NIPT, noninvasive prenatal testing.
Participants were asked to react to a series of statements regarding attitudes toward expanded NIPT by indicating whether they agree, disagree, or were unsure (-Table 6 ). Despite comfort level and utilization, a majority of participants $(91 \%, 77 / 85)$ agreed that practitioners need more information/education about expanded NIPT. This desire for more information is consistent across all groups of physicians, including those who reported that they have ordered expanded NIPT and those who have not. To further characterize the attitudes of obstetricians who were previously aware of the expanded testing option of NIPT, the responses were compared with those of individuals who were not previously aware of the expanded testing option of NIPT. Of those who were previously aware of the expanded testing option of NIPT, almost one-fifth $(19 \%, 12 / 64)$ agreed with the statement that it provides little added benefit. This was in comparison to those who reported that they were not aware of expanded NIPT, none of whom agreed that expanded NIPT provides a little added benefit $(p<0.05)$. In addition, out of the respondents who were aware of expanded NIPT, about half $(49 \%, 31 / 64)$ disagreed with a statement that the testing will replace invasive testing, whereas $18 \%$ (2/11) of those who were not aware of expanded NIPT disagreed with this statement $(p<0.01)$. Finally, $40 \%(25 / 64)$ of those who were aware of expanded NIPT agreed that it will become standard of care, whereas over half $(56 \%, 5 / 11)$ of those who were not previously aware of expanded NIPT agreed with this statement $(p<0.05)$.

Comfort level with explaining expanded NIPT was a significant predictor of respondents' attitudes toward expanded NIPT. Of those who were at least somewhat comfortable with explaining the expanded testing option of NIPT to patients, $37 \%$ (13/35) agree that expanded NIPT will replace invasive procedures. This is compared with just under one-quarter $(22 \%, 7 / 31)$ of those who reported being either somewhat uncomfortable or very uncomfortable with explaining the testing to patients who agreed with the statement that expanded NIPT will replace invasive testing $(p=0.03)$. Similarly, $41 \%(14 / 34)$ of those who are either somewhat comfortable or very comfortable explaining expanded NIPT to patients agreed with the statement that clinical utility and validity have been established for NIPT expanded testing. In contrast, only $7 \%$ (2/30) of those who are somewhat uncomfortable or very uncomfortable explaining expanded NIPT agree that clinical utility and validity have been established for expanded NIPT ( $p=0.03$ ).

The source from which obstetricians learn about the expanded testing option of NIPT is associated with whether they anticipate incorporating this testing option into their practice in the future. Although several participants selected multiple sources of information for learning about expanded NIPT, those who selected a single source of academic nature, including medical literature, a professional society/conference, a colleague, or an educational lecture, were compared with those whose sole source of information was marketing from laboratories performing the testing. Of those who selected a single source of information about expanded NIPT, the majority $71 \%$ (10/14) of those who had obtained 
Table 6 Obstetrician attitudes toward expanded NIPT $(n=85)$

\begin{tabular}{|c|c|c|c|c|}
\hline & $\begin{array}{l}\text { Agree } \\
(\%)\end{array}$ & $\begin{array}{l}\text { Unsure } \\
(\%)\end{array}$ & $\begin{array}{l}\text { Disagree } \\
(\%)\end{array}$ & $\begin{array}{l}\text { No response } \\
\text { (\%) }\end{array}$ \\
\hline The expanded NIPT option provides little added benefit & $12(14)$ & $30(35)$ & $41(48)$ & $2(3)$ \\
\hline $\begin{array}{l}\text { The conditions included in the expanded testing option were } \\
\text { chosen arbitrarily }\end{array}$ & $5(6)$ & $34(40)$ & $43(51)$ & $3(3)$ \\
\hline The expanded NIPT option will affect my practice & $29(34)$ & $28(33)$ & $25(29)$ & $3(4)$ \\
\hline The expanded NIPT option will replace invasive procedures & $25(30)$ & $23(27)$ & $35(41)$ & $2(2)$ \\
\hline Practitioners need more information/education about the test/technology & $77(91)$ & $3(3)$ & $2(2)$ & $3(4)$ \\
\hline Clinical utility and validity have been established for expanded NIPT & $19(22)$ & $47(55)$ & $15(18)$ & $4(5)$ \\
\hline NIPT expanded testing is going to simplify prenatal diagnosis & $32(38)$ & $33(39)$ & $15(17)$ & $5(6)$ \\
\hline Prenatal diagnosis of microdeletion syndromes reduces lifetime medical costs & $29(34)$ & $41(48)$ & $12(14)$ & $3(4)$ \\
\hline $\begin{array}{l}\text { The technology used in the NIPT expanded testing option is applicable to any } \\
\text { microdeletions/duplications in the genome }\end{array}$ & $11(13)$ & $48(56)$ & $22(26)$ & $4(5)$ \\
\hline The NIPT expanded testing option will eventually be standard of care & $30(35)$ & $45(53)$ & $6(7)$ & $4(5)$ \\
\hline
\end{tabular}

Abbreviation: NIPT, noninvasive prenatal testing.

information from only laboratory marketing were unsure of whether they would incorporate it into their practice in the future. In comparison, only $19 \%$ (5/26) of those who learned from only academic sources were unsure $(p<0.01)$.

\section{Discussion}

This study demonstrated that the majority of obstetricians surveyed were aware of the expanded NIPT option, yet few have actually utilized it in clinical practice. Not all physicians are comfortable talking with patients about the test and many overestimate its diagnostic ability. Physicians surveyed expressed a strong desire for more information regarding expanded NIPT.

This study revealed that one prominent source of information about expanded NIPT for physicians is marketing from laboratories that perform the test. While education from manufacturers, can benefit ordering physicians, and encourage them to learn more about prenatal testing options, currently available marketing materials from companies may not provide a full picture of the implications of this screening. For example, marketing pamphlets cite an up to $99.5 \%$ sensitivity and $>99 \%$ specificity for 22 q11 deletion syndrome, but they do not include information explaining that by factoring in a population prevalence of 1 in 2,000 , the positive predictive value is less than $5 \%{ }^{22}$ Although many obstetricians are receiving information about expanded NIPT from laboratories, participants in this study who had learned about expanded NIPT from academic sources were significantly more likely to plan to consider incorporating expanded NIPT into their practices in the future. The survey did not ask what sources physicians prefer to receive information from, but these results would support the hypothesis that physicians prefer to learn about the expanded testing option from academic sources, such as guidelines published by professional societies.
Although few professional guidelines specific to expanded NIPT have been published, guidelines for other types of screening can provide direction when new testing becomes available. A joint statement by the American College of Medical Genetics, the American College of Obstetricians and Gynecologists, the National Society of Genetic Counselors, the Perinatal Quality Foundation, and the Society for Maternal Fetal Medicine (SMFM) on expanded carrier screening cautions that if residual risk information has not been determined, laboratory reports should clearly communicate the limitations of interpretation of screening. ${ }^{23}$ This guideline calls upon laboratories and health care professionals to communicate the results of carrier screening for patients in a realistic manner. It is reasonable to think that discussion surrounding expanded NIPT should follow suit with the same urgency. Furthermore, the observed overestimation of expanded NIPT's accuracy and diagnostic ability parallels a finding from Benn et al, which reported that nearly half of obstetricians view traditional NIPT as a full substitute for invasive testing. ${ }^{24}$ However, the majority of physicians in this study reported making proper recommendations following a positive result, including offering invasive, diagnostic testing, and/or a referral to a specialist such as an MFM or a genetic counselor.

Limitations to this study include that participants may not have interpreted all questions as intended. This study population was limited by size, geography, and practice setting; therefore, it may be difficult to generalize these findings to obstetricians from other geographical areas and diverse practice settings. Despite this limitation, the physicians included in this study who attend meetings and take the initiative to fill out a survey may be more likely to be familiar with the expanded testing option and more motivated to learn more about it. This may suggest that these results underestimate the need and desire for physician education regarding expanded NIPT. 
It is preferable that patients receive accurate and appropriate information before testing for purposes of both informed consent and prevention of psychological harm. Knowledgeable pretest counseling is particularly pertinent to expanded NIPT due to the complex nature of the technology and the often unfamiliar information it can produce, a concept which supports the SMFM recommendation for pretest counseling by a health professional, such as a genetic counselor, and the International Society for Prenatal Diagnosis recommendation for inclusion of clinical significance information in expanded NIPT reports. ${ }^{20,21}$ The model in which comprehensive information is provided only following a positive result is consistent with a trend in which technological ability to perform tests surpasses understanding of its validity, utility, and best practices. For this reason, the education of health care professionals who provide information about expanded NIPT to patients is crucial. Practitioners must consider whether the ability to prenatally screen for subchromosomal abnormalities is sufficient basis for performing the testing.

In addition, this study reveals that while less than half of obstetricians report being at least somewhat comfortable explaining expanded NIPT to patients, they are aware of the gaps in their knowledge about this prenatal screening and unequivocally desire more information about it. As the participants were accessed through obstetrical departmental meetings, educational presentations by knowledgeable colleagues at these meetings may be a good starting point. Obstetricians are ordering the expanded NIPT option and will presumably continue to do so, and the development of further professional guidelines for utilizing expanded NIPT is increasingly vital. Health care professionals have a responsibility to educate themselves and others regarding the expanded NIPT option and other prenatal screens to ensure that patients receive optimal care.

\section{References}

1 Chen S, Lau TK, Zhang C, et al. A method for noninvasive detection of fetal large deletions/duplications by low coverage massively parallel sequencing. Prenat Diagn 2013;33(6):584-590

2 Palomaki GE, Kloza EM, Lambert-Messerlian GM, et al. DNA sequencing of maternal plasma to detect Down syndrome: an international clinical validation study. Genet Med 2011;13(11): 913-920

3 Palomaki GE, Deciu C, Kloza EM, et al. DNA sequencing of maternal plasma reliably identifies trisomy 18 and trisomy 13 as well as Down syndrome: an international collaborative study. Genet Med 2012;14(3):296-305

4 Bianchi DW, Platt LD, Goldberg JD, Abuhamad AZ, Sehnert AJ, Rava RP; MatErnal BLood IS Source to Accurately diagnose fetal aneuploidy (MELISSA) Study Group. Genome-wide fetal aneuploidy detection by maternal plasma DNA sequencing. Obstet Gynecol 2012;119(5):890-901

5 Norton ME, Brar H, Weiss J, et al. Non-Invasive Chromosomal Evaluation (NICE) Study: results of a multicenter prospective cohort study for detection of fetal trisomy 21 and trisomy 18 . Am J Obstet Gynecol 2012;207(2):137.e1-137.e8
6 Mazloom AR, Džakula Ž, Oeth $\mathrm{P}$, et al. Noninvasive prenatal detection of sex chromosomal aneuploidies by sequencing circulating cell-free DNA from maternal plasma. Prenat Diagn 2013; 33(6):591-597

7 Bianchi DW, Parker RL, Wentworth J, et al; CARE Study Group. DNA sequencing versus standard prenatal aneuploidy screening. N Engl J Med 2014;370(9):799-808

8 American College of Obstetricians and Gynecologists Committee on Genetics. Committee Opinion No. 545: Noninvasive prenatal testing for fetal aneuploidy. Obstet Gynecol 2012;120(6): 1532-1534

9 Wilson KL, Czerwinski JL, Hoskovec JM, et al. NSGC practice guideline: prenatal screening and diagnostic testing options for chromosome aneuploidy. J Genet Couns 2013;22(1):4-15

10 Benn P, Borell A, Chiu R, et al. Position statement from the aneuploidy screening committee on behalf of the board of the international society for prenatal diagnosis. Prenat Diagn 2013; 33(7):622-629

11 Bianchi DW, Wilkins-Haug L. Integration of noninvasive DNA testing for aneuploidy into prenatal care: what has happened since the rubber met the road? Clin Chem 2014;60(1):78-87

12 Jensen TJ, Dzakula Z, Deciu C, van den Boom D, Ehrich M. Detection of microdeletion $22 \mathrm{q} 11.2$ in a fetus by next-generation sequencing of maternal plasma. Clin Chem 2012;58(7):1148-1151

13 Peters D, Chu T, Yatsenko SA, et al. Noninvasive prenatal diagnosis of a fetal microdeletion syndrome. N Engl J Med 2011;365(19): 1847-1848

14 Srinivasan A, Bianchi DW, Huang H, Sehnert AJ, Rava RP. Noninvasive detection of fetal subchromosome abnormalities via deep sequencing of maternal plasma. Am J Hum Genet 2013;92(2): 167-176

15 Yu SC, Jiang P, Choy KW, et al. Noninvasive prenatal molecular karyotyping from maternal plasma. PLoS ONE 2013;8(4): e60968

16 Jia Y, Zhao H, Shi D, et al. Genetic effects of a 13q31.1 microdeletion detected by noninvasive prenatal testing (NIPT). Int J Clin Exp Pathol 2014;7(10):7003-7011

17 Rampášek L, Arbabi A, Brudno M. Probabilistic method for detecting copy number variation in a fetal genome using maternal plasma sequencing. Bioinformatics 2014;30(12):i212-i218

18 Zhao C, Tynan J, Ehrich M, et al. Detection of fetal subchromosomal abnormalities by sequencing circulating cell-free DNA from maternal plasma. Clin Chem 2015;61(4):608-616

19 Wapner RJ, Babiarz JE, Levy B, et al. Expanding the scope of noninvasive prenatal testing: detection of fetal microdeletion syndromes. Am J Obstet Gynecol 2015;212(3):332.e1-332.e9

20 Society for Maternal-Fetal Medicine (SMFM) Publications Committee. \#36: Prenatal aneuploidy screening using cell-free DNA. Am J Obstet Gynecol 2015;212(6):711-716

21 Benn P, Borrell A, Chiu RW, et al. Position statement from the Chromosome Abnormality Screening Committee on behalf of the Board of the International Society for Prenatal Diagnosis. Prenat Diagn 2015;35(8):725-734

22 Natera, Inc. Panoramatest. Available at: http://www.panoramatest.com/healthcare-provider. Accessed March 26, 2015

23 Edwards JG, Feldman G, Goldberg J, et al. Expanded carrier screening in reproductive medicine-points to consider: a joint statement of the American College of Medical Genetics and Genomics, American College of Obstetricians and Gynecologists, National Society of Genetic Counselors, Perinatal Quality Foundation, and Society for Maternal-Fetal Medicine. Obstet Gynecol 2015;125(3):653-662

24 Benn P, Chapman AR, Erickson K, et al. Obstetricians and gynecologists' practice and opinions of expanded carrier testing and noninvasive prenatal testing. Prenat Diagn 2014;34(2):145-152 